\title{
Can Artificial Intelligence be a Solution for the Challenges Faced by Indian Health Care?
}

\author{
Buddhdev Pandya MBE \\ Policy Director, British Association of Physicians of Indian Origin; \\ Ex-Editor, Asian Age \\ buddhdevp@gmail.com
}

cite as: Pandya B. Can Artificial Intelligence be a solution for the challenges faced by Indian Health Care? Sushruta 2019 (Nov) 30-32 DOI: 10.38192/12.1.17

India with a population of nearly 1.3 billion has unimaginable challenges in planning for a health care system. While accessibility in geographical terms is challenging for a huge country, the rich and affluent can access state of the art services, while lower- and middle-income population are left to face cruel choices, as the state health infrastructure remains sparse and grossly under-funded. The evolution of new quantum computing and its capitulation with robotic or molecular technologies is creating faster diagnostic and treatment algorithms. With the availability of satellite communications for remote areas and advances in accurate sensing gadgets, the possibility of providing rapid healthcare via artificial intelligence is becoming closer to reality.

There is an emerging paradigm shift in how health conditions are diagnosed and treated. Advances with portable and simple to use medical devices/technologies may provide the answer in reaching-out to communities living in geographically remote and economically deprived areas. One of the main challenges of India's health care provision has been severe limitation of financial resources. This is further compounded by many others such as waste of resources, limited training opportunities for healthcare staff and ineffective delivery of plans.

A culture change of policy towards 'patient- centric solutions' is needed if Indian health care is to take advantage of the new opportunities that innovations in artificial intelligence (AI) has to offer. In the search for answers, India must first integrate affordability as well as accessibility when addressing healthcare challenges!

The net worth of the Indian health care sector is expected to rise threefold from US\$110 billion in 2016 to US\$372billion in 2022. This growth driven by medical tourism, a burgeoning hospital sector, and increased affluence of a segment of the population, makes India an attractive prospect for medical device companies looking for partnerships. The Indian medical device industry has an opportunity to leapfrog innovations combining physical devices and integrated digital networks for far reaching benefit. 
Indian in-vitro diagnostics (IVD) market consists of many different segments. There is an urgent need for affordable, portable, easy-to-use solutions in low-resource settings. The growth of the medical device industry is largely driven by the level of the healthcare expenditure, advances in technology and the need to serve an ageing population with chronic diseases. For India with diverse inequalities in accessing basic healthcare, Al offers a unique opportunity. The health care industry presents a gap in trained clinicians and inadequate infrastructure that is compounded by low government spending. Yet, it a country with the most room for innovative, sustainable and scalable healthcare technology solutions. In its last report (2019) telecom regulator revealed that India has 1.16 billion mobile subscribers, a notable penetration. This is indeed one of the most vantage points where the population equipped with smartphones can access digital technologies.

We are witnessing a surge of smart wearable (wristwatches) devices that can track vital signs and alert the wearer/ provider, when the condition reaches set parameters. In developed nations, many aspects of remote monitoring of patient's wellbeing and treatment outcomes are already established. The rise in diabetes, heart and kidney disease in addition to the traditional scourge of infectious diseases provides a fertile substrate for Al solutions.

In February 2018, the Government of India announced that 1,50,000 Health \& Wellness Centres (HWCs) would be created by transforming existing Sub Health Centres and Primary Health Centres to deliver Comprehensive Primary Health Care and declared this as one of the two components of Ayushman Bharat. These centres would benefit from a network for the integration of information; managing patients and their follow-up using Al. In local health planning such information would be invaluable and mobile units would be able to reach remote areas powered by portable technology.

The Ayushman Bharat - National Health Protection, also intends to provide support for secondary and tertiary care hospitalisation for many poor and vulnerable families. A welldesigned Al algorithm would help improve efficiency and provide basic healthcare to a larger cohort. India has a history of embracing 'pilot schemes' of public-private partnerships that appear to be successful but eventually, few of them have been scaled up to meet India's health challenges. In the last decade products for tuberculosis medication adherence monitoring and vital parameter monitors in the primary healthcare places with the telemedicine programs medical expertise has been providing without doctors the doctor being present. Al applications being developed and deployed in India include algorithms that analyse chest $x$-rays and other radiology images, read ECGs and spot abnormal patterns, automatically scan pathology slides and assess fundus photographs for signs of retinopathy.

In many countries, the healthcare systems are often slower to adopt change than their counterparts in other industries and insist on a higher rigour of testing for patient safety. But in India, it is not only regulation that stifles innovation. Most healthcare is provided by the 
private sector and paid for by the individual, hence affordability is huge challenge in adoption of new technologies.

Already in the USA Outcome-Based Contracts (OBC) are being implemented which is proposed as a measure to award innovation, based on the actual performance of treatments and interventions in real patients. This can be challenging as outcomes are often difficult to measure or quantify. Al has the potential to fill this gap. Developments in this area may support reimbursements under the proposed 'Modi Care scheme' (ref) consisting of an insurance cover for the poor.

- India needs a leap through innovative, sustainable and scalable Al technologies with the potential to improve outcomes through remote access, screening, prevention, and treatment for the deprived segments of population.

- The private providers and cutting-edge innovators need to be incentivised in shaping the advanced medical sector that India needs to attract medical tourism.

- Al and integration of outcomes data can support a culture shift towards patient safety and restore trust in the healthcare system. 